\title{
Wirtschaften im Zusammenhang: Sorge, Sympathie, Sinn
}

Gabriele Sorgo

Als wir das vorliegende Projekt konzipierten, lag unser Interesse auf dem Zusammenspiel des persönlichen Engagements und des Arbeitseinsatzes bäuerlicher Akteur*innen einerseits mit den regionaltypischen sozialen und ökologischen Ressourcen andererseits. Als zentrale Theoriemodelle für die Makroanalyse agrarsoziologischer Daten hatten wir Karl Polanyis Theorie der "Einbettung « ${ }^{1}$ und das Konzept der »Gabe« von Marcel Mauss ${ }^{2}$ gewählt. Denn menschliche Zuwendungen an Zeit, Aufmerksamkeit und materiellen Gaben repräsentieren soziale Beziehungen, betten also ein, und überschreiten die Grenzen zwischen Menschen, Tieren und Dingen, die gewöhnlich als isoliert betrachtet werden. Zuwendung erzeugt Beziehung - und Beziehung überschreitet Grenzen, auch jene zwischen den Spezies. Im Verlauf der Erhebungen wurde diese theoretische Basis um die Begriffe der Resilienz ${ }^{3}$ und der Relationalität erweitert. Die Studien von Ika Darnhofer stellen hierzu im Bereich der Agrarsoziologie die bei weitem spannendsten und interdisziplinär anschlussfähigsten Forschungsansätze dar, denen wir viel verdanken. ${ }^{4}$

Ziel unseres Vorhabens war es, zu zeigen, dass auch Geräte, Landschaften und Pflanzen von Menschen in ein lebensweltliches Be-

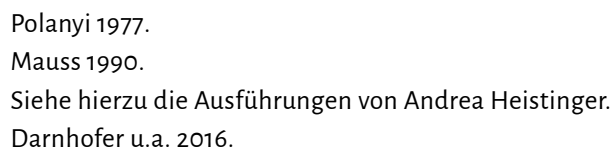


ziehungsgeflecht eingewoben werden, das nur zu einem geringen Teil nach marktwirtschaftlichen Kriterien funktioniert und wohl gerade deshalb die Grundlage des guten Lebens ${ }^{5}$ darstellt. Diese Gedanken sind keineswegs neu, sondern finden sich schon des längeren in modernen ökonomischen Theorien, die der profitorientierten Marktwirtschaft dienen sollen, wenn auch bisher ohne Einbindung der Tierund Pflanzenwelt. ${ }^{6}$ Eine Evaluierung so genannter »weicher Faktoren « des unternehmerischen Erfolgs, wie von Organisationsentwickler*innen oft empfohlen, stellt jedoch keinen Interessensschwerpunkt dieser Studie dar. ${ }^{7}$ Vielmehr gehen wir der Frage nach, inwieweit die Motivation ökologisch wirtschaftender Bauern und Bäuerinnen für ihre durchaus riskanten betrieblichen Entscheidungen sich aus ihren biographischen Erfahrungen und ihren Familiengeschichten herleitet. Jedes menschliche Individuum wird in Beziehungen hineingeboren und während des Heranwachsens von ihnen geprägt. Verknüpft man Stephen Gudemans wirtschaftsanthropologische Perspektive einer stets vorhandenen Verschränkung profitorientierter mit sozial motivierten Austauschprozessen ${ }^{8}$ zusätzlich mit der artenübergreifenden relationalen Ontologie von Donna Haraway, so wird das hegemoniale, profitorientierte Theoriemodell der Ökonomie gewaltig relativiert. Eine andere, humanere Perspektive eröffnet sich, die jedoch die herkömmlichen betriebswirtschaftlichen Zugriffe auf die Wirklichkeit nicht erkennen und auch nicht anerkennen wollen. Seit drei Jahrzehnten fordert Haraway mit ihrem Aufruf zum artenübergreifenden Denken angesichts des bedrohten Planeten mehr Demut ein. ${ }^{9}$ Sie weist darauf hin, dass sämtliche Beziehungen, soziale wie biologische, von denen und in denen ein Individuum ausschließlich leben kann, der Existenz menschlichen Lebens vorausgehen. Sich die Menschen nur als abgeschlossene, selbstbezogene Systeme vorzustellen, betont Haraway,

\footnotetext{
$5 \quad$ Nussbaum 1999, S. 57 f.

6 Vgl. Krippner u.a. 2004.

7 Vgl. Schönborn 2008, S. 100.

8 Gudeman 2016.

9 Haraway 2018.
} 
mache blind für die Tatsache, dass wir unsere Existenz sowohl vergangenen als auch gegenwärtigen Beziehungen und Kooperationsformen mit unzähligen Lebewesen verdanken. ${ }^{10}$ Einige dieser Kooperationsformen sind dem Feld der Ökonomie zuzuordnen, die meisten müssen jedoch unter anderen Gesichtspunkten betrachtet werden. Zusammen bilden sie ein Bündel an Relationen, das Menschen und ihre Wirtschaftsformen als relationale Phänomene begreifbar macht. Die Erforschung des menschlichen Mikrobioms relativiert z.B. Vorstellungen von einem klar gegenüber seiner Umwelt abgegrenzten menschlichen Organismus. Vielmehr sind Menschen Holobionten ${ }^{11}$ : Eine Versammlung von Milliarden von Kleinstlebewesen, die zusammenarbeiten und sich austauschen, damit ein menschlicher Stoffwechsel tadellos funktioniert. ${ }^{12}$ Bakterien des Verdauungstraktes, ihre Vielfalt und ihre Wechselwirkungen beeinflussen sogar die menschliche Psyche. ${ }^{13}$ So gesehen ist es dringend notwendig, auch in sozialwissenschaftlichen Forschungen den Bodenbakterien, die unsere Lebensmittel wachsen lassen, mehr Aufmerksamkeit zu schenken. Genau das machen die von uns interviewten Bauern und Bäuerinnen. Das einst Bedeutungslose und durch disziplinäre Grenzen Aussortierte, rückt nun endlich in den Fokus der Forschung. Die Wissenschaften benützen große Theorien, um die Komplexität des Lebendigen gedanklich erfassen zu können. »Profitorientierung« oder "Subsistenzorientierung « erscheinen dann als zwei diametral entgegengesetzte Wirtschaftsformen, die anhand von klar definierten Merkmalen unterschieden werden. Doch diese Dramatisierung der Differenz kann zu falschen Ergebnissen führen. Forschung sollte zwar immer theoriegeleitet stattfinden, doch die Theorien dürfen für Widersprüche und ambivalente Ergebnisse, die sich nicht so einfach in die erstellten Kategorien einfügen lassen, nicht blind machen. Das reale Leben der Menschen ist weitaus komplexer.

\footnotetext{
10 Haraway 2003, S. 21.

11 Grassberger 2019, S. 200f.

12 Collen 2015.

13 Valles-Colomer u.a. 2019.
} 


\section{Geben, Nehmen, Werden}

Mit dem Wissen, dass menschliche Individuen sich ihren sozialen und materiellen Beziehungen verdanken, erscheinen auch vermeintlich banale Interaktionen in einem anderen Licht. Man gibt den Blumen Wasser und schenkt den Nachbarskindern Himbeeren oder einem Besucher ein paar gute Tipps für den eigenen Garten. Der Alltag besteht aus vielen solchen kleinen Geschenken, die unter Menschen Verbindlichkeit und Vertrauen erzeugen. Unsere ursprüngliche Hypothese lautete, dass naturnah wirtschaftende Betriebe diese nicht-gewinnmaximierenden Handlungen häufiger einsetzen als mehr agrarindustriell ausgerichtete und dass sie deswegen auch der Region statt nur dem eigenen Unternehmen Gewinn bringen. Die derzeit vorherrschende agrarindustrielle Nutzung von Boden und Tieren ist eine Wirtschaftspraxis, die auf Dauer zu viel »nimmt « und sowohl den Boden und die Artenvielfalt als auch die sozialen Zusammenhänge schädigt. Die Agrarindustrie folgt einem modernen marktrationalen Konzept von Landbau, das sich aus einigen wenigen Parametern (Bodenqualität, Klima, Dünger, Maschineneinsatz, Wasser, Lagerung, Transport) zusammensetzt, die so austariert werden, dass sie die Quantität an Lebensmitteln kurz- und mittelfristig erhöhen, langfristig jedoch gefährden, wie der Weltagrarbericht von 2008 darlegen konnte. ${ }^{14}$ Dabei leben in einer Hand voll fruchtbarem Humus mehr Mikroorganismen als Menschen auf der Erde. Die Beziehungen und Wechselwirkungen zwischen ihnen sind noch lange nicht ausreichend erforscht, obwohl das Überleben der Menschheit von ihnen abhängt.

Auf der Suche nach kurzfristigem Gewinn reduziert die erdölbasierte, hochtechnologische Landwirtschaft langfristig die Mikroorganismen des Bodens und lässt die Fruchtbarkeit sinken. Die kultivierten Pflanzensorten werden auf einige wenige hochgezüchtete Sorten reduziert. Parallel mit der Zunahme der Monokulturen verließen die jungen Menschen im 20. Jahrhundert das Land (und tun es bis heute). Die verbleibenden Bauern und Bäuerinnen wurden häufig aufgrund

VgI. IAASTD 2008. 
der marktwirtschaftlich erzwungenen Rationalisierungen zu hochverschuldeten Ressourcenlieferanten eines weltweiten Agrarsystems. Ihre Beziehungen zum Boden, zu den Tieren und auch zu den Menschen, für die sie Lebensmittel erzeugen, können Agrarunternehmer*innen heute nicht mehr selbst bestimmen, sondern sie unterliegen den Auflagen des globalen Nahrungsregimes. ${ }^{15}$ Um die Unterschiede zwischen agrarindustrieller und biobäuerlicher Herangehensweise wissenschaftlich differenzierter $\mathrm{zu}$ erfassen, ist es aus unserer Sicht unbedingt angebracht, soziologische und anthropologische Theorien auf den Umgang mit Pflanzen und Tieren auszudehnen. Menschen sind nicht nur soziale, sondern im umfassenden Sinn relationale Wesen, sie sind eher Prozesse, die durch Beziehungen ins Leben gerufen und am Leben erhalten werden. ${ }^{16}$ In den euroatlantischen Industriegesellschaften haben Menschen aber unter dem Einfluss kapitalistischer Produktions- und Konsumbedingungen die Gewohnheit angenommen, sich die Beziehung zu sich selber und zur Umwelt nach dem Muster eines neutralen Erfassens von dinglichen Gegebenheiten vorzustellen. ${ }^{17}$ Daraus resultiert eine Handlungspraxis, die selbst verdinglichte Gestalt annimmt. Bäuerliches Handeln wurde oft $\mathrm{zu}$ teilnahmslosem Handeln. ${ }^{18}$ Früchte werden aufgrund von Verträgen mit Handelspartner*innen $\mathrm{zu}$ früh geerntet und Jungtiere von den Muttertieren getrennt, weil die Logik des Marktes dies verlangt. Die zahlreichen bloß kalkulatorischen Tauschprozesse des Alltags prägen auf Dauer die Deutungsgewohnheiten der Menschen moderner Industriegesellschaften, sodass sie die lebenswichtigen Beziehungen zur Umwelt ausblenden. Nichtsdestotrotz müssen Menschen sich als

Der Begriff food regime stammt von Harriet Friedman und Philipp McMichael (1989). Van der Ploeg (2018b, S. 245) bevorzugt food empires und definiert sie so: »Food empires are globally operated networks with clear centres of command. They control large, and expanding parts of the production, processing, distribution and consumption of food, although they are not necessarily involved in the physical realities associated with these processes.«

16 Vgl. Donati 2011.

17 Vgl. Honneth 2005, S. 36.

18 Vgl. Müller 1998, S. 167f. 
soziale Wesen dennoch stets um gelingende materielle und soziale Austauschprozesse mit der Umwelt bemühen. Das Feld der dafür notwendigen Interaktionen muss aber heute angesichts des botanischen, zoologischen, psychologischen und anthropologischen Wissens unbedingt weiter gefasst werden als derzeit in der Agrarsoziologie üblich. ${ }^{19}$ Nahrung - Erzeugung, Herstellung und Verzehr - gilt nach Mauss als soziales Totalphänomen ${ }^{20}$, weil sie menschliche Belange in materieller, rechtlicher, wirtschaftlicher, religiöser, ästhetischer und sozialer Hinsicht beeinflusst. Sich Ernähren, der Akt der Konsumption, stellt eine Interaktion auf vielen Ebenen dar. Ein durchschnittlicher Körper beherbergt vor allem im Darmtrakt unzählige Lebewesen, die sich von ihrem Wirt unterscheiden und ein eigenes Leben führen. Wo endet ein Körper und wo fängt er an? Das hochentwickelte Gehirn kann uns nicht davor bewahren, dass wir essen müssen und dabei zugleich auf vielfältige Weise von unserer Umwelt vereinnahmt, durchdrungen und verbraucht werden. Dieses Wissen um die menschliche Leiblichkeit, die mehr ist als nur ein Körper, hat Akteure*innen in der Landwirtschaft immer wieder dazu angespornt, agrarindustrielle Produktionsstile abzulehnen. Die interviewten Akteur*innen dieses Forschungsprojektes leben in spezifischen familiären, regionalen, sozialen, ökologischen und wirtschaftlichen Beziehungsgeflechten und zeichnen sich dadurch aus, dass sie diese Einbettung auch reflektieren. Weit entfernt von jeder Sozialromantik tauchen immer wieder Wendungen in ihren Erzählungen auf, die dies zum Ausdruck bringen. Sie wissen, dass sie in Food webs ${ }^{21}$ leben und haben Respekt davor.

\footnotetext{
19 Vgl. Darnhofer u.a. 2016.

20 Mauss 1990, S. 17-18.

21 Alle Lebewesen befinden sich in Food webs, die aus der Verknüpfung von Nahrungsketten bestehen und den komplexen Zusammenhang und das Miteinander-Verbunden-Sein auf diesem Planeten repräsentieren. Vgl. Puig de la Bellacasa 2017, S. 69-94.
} 


\section{Austausch, Abhängigkeiten, Einbettung}

Die deutlich spürbaren Folgen des Klimawandels schaffen es gegenwärtig zum ersten Mal in der Geschichte, dass sich breite Bevölkerungsschichten über die Folgen der modernen Produktionsbedingungen den Kopf zerbrechen. Die Mondlandung vermittelte 1969 distanzierte Bilder des Planeten Erde, die damals wohl zum ersten Mal als gemeinsamer Lebensraum aller Menschen vorstellbar wurde. Nun scheint es, dass der Klimawandel dieses Bewusstsein sinnlich spürbarer macht. Denn er vermittelt nicht die Souveränität, dass die Menschen sich über das Irdische erheben könnten, sondern unterwirft sie eher den Naturvorgängen und lässt den Zusammenhang auch als Abhängigkeiten fühlbar werden. Das kann eine Chance für eine gesellschaftliche Transformation sein. Um die Qualität der materiellen, sozialen und symbolischen Eingewobenheit der Menschen so $\mathrm{zu}$ beschreiben, dass auch neue Dimensionen der umweltlichen Einbettung sichtbar werden, wählte Haraway in ihrer jüngsten Publikation ${ }^{22}$ das Wort "sympoietisch «, das die Umweltwissenschaftlerin Beth Dempster ${ }^{23}$ vorgeschlagen hat. Dempster wollte mit ihrer Wortschöpfung aus den griechischen Begriffen sym - »mit« - und poiesis - „Erschaffung«, die Abhängigkeiten und die Austauschprozesse zwischen lebenden Organismen ins rechte Licht rücken. Denn alle Lebewesen dieses Planeten existieren miteinander und es gibt mehr, das sie verbindet, als trennt. Die großen klassischen Theorien verlocken dazu, die alltäglichen Mikroprozesse arbeitender Menschen entlang von Kategoriensträngen wie Natur/Kultur, Mensch/Tier, Arbeit/Spiel, Produktion/Reproduktion, Stadt/Land, männlich/weiblich usw. zu sortieren. Für eine am (Über-)Leben orientierte Wirtschaft wäre es aber besser, sämtliche ökonomisch relevanten Handlungen als sozial und regional sowie zeitlich gebundene Strategien zur Bestreitung des je individuellen Lebensunterhaltes zu erfassen, welche diese Dichotomien der analytischen Wissenschaften ständig überschreiten. Darauf hat die feministisch-ökonomische Geografin

22 Haraway 2018, S. 47-83.

23 Dempster 1998. 
J. K. Gibson-Graham nachdrücklich hingewiesen. ${ }^{24}$ Die Erhebungen in der Oststeiermark waren konzeptuell von vorneherein vom Widerspruch zwischen den Ansprüchen des ökologischen Landbaus und den niedrigen Preisen der agrarindustriellen Konkurrenz geprägt. Wie können Betriebe in diesem Widerspruch Wirtschaftsstile entwickeln, die umweltschonende Arbeitspraktiken mit angemessenen Preisen verbinden? Betriebe müssen sich im ökonomischen Umfeld bewähren und verfügen über je völlig unterschiedliche Böden, Menschen und finanzielle Rücklagen. Gibson-Graham schlägt vor, näher bei den erhobenen Daten zu bleiben und die "starken « Theorien nur vorsichtig an das Datenmaterial anzulegen. Der Alltag wirtschaftender Menschen, meint sie, ist eine »chaotische Landschaft ${ }^{25}$ aus verschiedensten Praktiken, an deren Komplexität Theorien herangetragen werden, die den Weizen von der Spreu trennen helfen sollen. Dies mag der besseren Kommunikation unter Wissenschaftler*innen dienen, unterbindet aber das Aufbrechen ihrer Denktraditionen. Wenn eine Biobäuerin ihren Kräuterfrischkäse zehn Jahre lang an ein Fünfsternehotel liefert, heißt das noch lange nicht, dass sie ihren Betrieb agrarindustriell strukturiert und dass ihr Vorgehen auch allen anderen Käseproduzent*innen als Strategie weiterempfohlen werden sollte. Das Moment der Flexibilität und das unvorhersehbare taktische Vorgehen so genannter resilienter Betriebsführer*innen geraten aus dem Blick. Theoretische Scheuklappen können dazu verleiten, das Einmalige und Unnachahmliche zu übersehen. Gerade die so genannten »neuen Bauern und Bäuerinnen ${ }^{26}$ stellen keine einheitliche Gruppe in Europa dar, obwohl zahlreiche agrarsoziologische Studien das vermitteln wollen und einen Kriterienkatalog dazu entwickelt haben. ${ }^{27}$ Diese Studien liegen nicht falsch, folgen jedoch den klassischen Vorgangsweisen der Soziologie, die eine Typenbildung vornimmt, die vielleicht angesichts eines derart raschen Wandels ökonomischer Bedingungen und angesichts großer

\footnotetext{
24 Gibson-Graham 2014.

25 Gibson-Graham 2014, S. 148.

26 Van der Ploeg 2018b.

27 Milone \& Ventura 2019; Van der Ploeg 2018b.
} 
regionaler Unterschiede wenig verlässliche Aussagen über die Zukunft bäuerlicher Betriebe bietet. Die agrarsoziologischen Forschungen der 1990er Jahre lieferten z.B. eher pessimistische Ergebnisse vom »Niedergang der lokalen Ökonomien ${ }^{28}$ und gingen von einer intrinsischen Verbindung traditioneller Bauerngesellschaften mit lokalen Ökonomien aus. Christa Müller schilderte in ihrer ausgezeichneten Studie den Mentalitätswandel bäuerlicher Produzent*innen, die ihr traditionelles umwelt- und suffizienzorientiertes Denken zugunsten einer profitorientierten Anschauung aufgaben. Müller unterscheidet zwischen einer Außenwirtschaft und einer Innenwirtschaft der bäuerlichen Familienbetriebe und kommt zu dem Schluss, dass die erzwungene Abhängigkeit vom Weltmarkt letztlich die noch verbleibende Subsistenz - die Innenwirtschaft - »entökonomisierte ${ }^{29}$ Damit meint sie, dass diese Innenwirtschaft mehr oder minder zu einem Hobby der Bäuerinnen wurde. Doch mit ihrer Wortwahl hat sie den Denkstil der modernen Betriebswirtschaftslehre übernommen und die dörfliche Realität der wirtschaftswissenschaftlichen Dichotomie bezahlt/unbezahlt unterworfen. Zwischen den Eigenschaftswörtern bezahlt und unbezahlt erstreckt sich ein Raum von Möglichkeiten, die erst benannt werden müssen. Um wesentliche Beiträge zum Gemeinwohl nicht unsichtbar werden zu lassen, ist es notwendig, die wissenschaftliche Beschreibung der Realität anzupassen statt umgekehrt. Sonst wird mit der Übernahme dieser vereinfachenden Dichotomie einmal mehr unsichtbar, dass die Subsistenzwirtschaft ohne finanziellen Profit gleichwohl einen bedeutenden ökonomischen Beitrag leistet, wie feministische Theoretikerinnen schon seit den 1960er Jahren darlegen. ${ }^{30}$ Die Begriffe der rein profitorientierten Betriebswirtschaft erwecken den Eindruck, dass die häusliche Produktion nichts zum Auskommen beiträgt, weil im Supermarkt billigere Konfitüren, Suppen und Würste angeboten werden. Dies entspricht jedoch nicht der Wahrheit. Häusliche Produktion mag kein Geld einbringen, sie kann es aber 
sparen, außerdem ist sie sozial und symbolisch wirksam und sorgt für eingebettete Nahrungsmittel, die Hersteller*innen ebenso wie Essende in Beziehung setzen und mit Sinn versorgen. Solche Beziehungen stellen die Grundlage jeder funktionierenden Marktwirtschaft dar. Werbung für Markenwaren versucht z.B. genau diese Einbettung so manchen industriell erzeugten Produkten künstlich hinzuzufügen. Bezeichnungen wie »Mit Liebe gekocht« oder »Nach Großmutters Rezept« oder »Ein gutes Stück Heimat« etc. simulieren nicht vorhandene Beziehungen und sollen bei den Konsument*innen Vertrauen erzeugen. Diese Werbungen beweisen, dass es um Werte geht, die ökonomisch relevant sind. Menschen brauchen diese sinnstiftenden Beziehungen ebenso wie pestizidfreie Feldfrüchte. Daniel Miller hat daher sogar die Einkaufstätigkeiten und das Auswählen der Waren als kulturschaffende Tätigkeiten bezeichnet, weil dabei aus vereinzelten fremden Dingen Bausteine für das soziokulturelle Konstrukt "Haushalt « entstehen, das sinnstiftend wirkt. ${ }^{31}$ Erst recht gilt das für häusliche Nahrungsmittelherstellung. Übertragen auf die bäuerlichen Betriebe heißt das, dass in der Spätmoderne nun auch die unbezahlte häusliche Herstellung von Lebensmitteln, die in der klassischen Wirtschaftstheorie bis in die 1960er Jahre der Reproduktion der bezahlten Arbeitskräften $\mathrm{zu}$ dienen hatte, dabei aber noch sichtbar war, nun in unsichtbare Reproduktionsarbeit verwandelt wurde und dadurch gemäß dieser Theorie nicht mehr zu ökonomisch verrechenbaren Tätigkeiten zählte. ${ }^{32} \mathrm{Ab}$ den 1970er Jahren verlor die Rolle der Hausfrau ihre gesellschaftliche Anerkennung und die »doppelte Vergesellschaftung « ${ }^{33}$ der Frau wurde in allen Schichten zur Selbstverständlichkeit. Doch eine Wirtschaftstheorie, die Natur und Hausarbeit als unendlich verfügbare Güter voraussetzt, die einerseits nichts wert sein sollen, während die Wirtschaftsleistung doch andererseits ohne sie nicht

$31 »[$...] consumption is used to extract culture as the selfconstruction of humanity from the intractable but essential institutions of the modern world, such as the market or bureaucracy.« Miller 1995, S. 34.

32 Kosnik 2019 und 2018.

33 Becker-Schmidt 2003. 
zustande käme, ist natürlich falsch. ${ }^{34}$ Seit den 1970er Jahren ignorieren neoliberale Wirtschaftstheorien sowohl Subsistenzarbeit als auch Konsumarbeit völlig. Dies führt einerseits zu steigenden Bedürfnissen nach regionalen Lebensmitteln - weil Lebensmittel eben doch mehr sind als nur Kalorien mit Geschmack - doch andererseits auch zu steigenden Arbeitszeiten auf naturnah wirtschaftenden bäuerlichen Betrieben, die jetzt mit niedrigen Weltmarktpreisen mithalten und zusätzlich unbezahlte affektive Arbeit leisten müssen. Affektive Arbeit bindet unter industriekapitalistischen Bedingungen die mehr oder minder fremden Waren und zwar ganz besonders Lebensmittel in sinnstiftende Beziehungsnetze ein und hilft, sie in die Weltauslegung und in die Lebenswelten der Konsument"innen $\mathrm{zu}$ integrieren. Die erfolgreichen Biomarken »Ja Natürlich« und »Zurück zum Ursprung«, die in Österreich von Supermarktketten entworfen wurden, werben mit Beziehungen. Fleisch, Eier und Milchprodukte werden mit ihrer Einbettung, d.h. mit Herkunftsnamen und Bildern ihrer Produzent*innen in idyllischer Natur verkauft. Der Anteil unbezahlter Arbeit, die letztlich "aus Liebe« zum Boden (ohne nationalistische Ideologie) und $\mathrm{zu}$ den essenden Mitbewohner*innen verrichtet werden, ist jedoch auf Biobetrieben sehr hoch. Denn Biobetriebe, die an große Handelsketten liefern, unterliegen denselben Wettbewerbsgesetzen wie konventionelle Betriebe und daher setzt der Markt sie deutlich stärker unter Druck, möglichst billig zu produzieren. So wird der ursprüngliche Gedanke des Wirtschaftens im Einklang mit der Natur durch die Gesetze des Marktes pervertiert. Biomonokulturen und Biomasttierzuchtanstalten entstehen. Sorgsame Landwirtschaft verwehrt sich diesen Vertriebsschienen, steht aber dennoch in Konkurrenz mit

34 Die häusliche Arbeit der Frauen fand bis in die 1960er Jahre Anerkennung. Lohn wurde jedoch vorrangig den Männern als »Familienernährern « ausgezahlt, um das Machtgefälle zwischen den Geschlechtern aufrecht zu erhalten. Der Spätkapitalismus verleugnet den Wert häuslicher Subsistenzarbeit und Sorgearbeit vollends und verdrängt die Geschlechterfrage, während er die Frauen (durch niedrige Löhne und unbezahlte Sorgearbeit) doppelt ausbeutet. Vgl. Soiland 2016. 
billigen Lebensmitteln. Der persönliche Einsatz und die Bereitschaft, je nach Saison sehr viel zu arbeiten sind hier sehr hoch. Die moderne Betriebswirtschaftslehre hält jedoch weiter daran fest, dass unbezahlte Arbeiten für Menschen, Regionen und Nahrungsmittelqualität keine Rolle spielen. Da heute jedoch belegt werden kann, dass Ökonomie nicht auf das Feld der geldbasierten Austauschprozesse beschränkt ist, verdeckt ein Begriff wie »Entökonomisierung« im Grunde, dass es sich um willkürliche kulturelle Entscheidungen handelt, was bezahlt wird und was nicht. Denn wirtschaftliches Handeln wird hier auf eine einzige ökonomische Theorie reduziert, die demnächst den Planeten in einen Kollaps zwingen könnte. Umso wichtiger ist es, diese mächtige Theorie, die behauptet, dass es bei erfolgreichen Betrieben nur um rationales Kalkül und finanziellen Profit ginge, zu entthronisieren. Die Organisationsentwicklung hat längst vorgezeigt, wie sehr der Bestand von großen Firmen von deren sozialen Klimata und Firmenkulturen abhängen kann. ${ }^{35}$

Im agrarsoziologischen Forschungsfeld wies Robert Hettlage schon 1989 darauf hin, dass bäuerliches Wirtschaften von Theoretiker*innen häufig als konservativ beharrend und als irrational dargestellt würde. ${ }^{36}$ Dies ist ein Schicksal, das Bauern und Bäuerinnen mit den Hausfrauen teilen. Doch solche Aussagen bringen die mittlerweile stark kritisierte Überheblichkeit von Wissenschaftler*innen zum Ausdruck, die das Modell des modernen Wirtschaftskapitalismus niemals zu hinterfragen gelernt haben. Die ökonomischen Diskurse des 20. Jahrhunderts ignorierten die Abhängigkeit der industriellen Expansion und des Finanzkapitalismus von der Subsistenzwirtschaft jeglicher Art, sei sie bäuerlich oder haushälterisch. Allein Kenneth Galbraith hat schon sehr früh mit seinem Begriff der »Konsumarbeit « nachdrücklich darauf hingewiesen, dass die Industrie ihre Gewinne der unbezahlten, meist weiblichen Arbeit in Haushalten verdankt, das heißt also der weitgehend unbeachtet gebliebenen, völligen sozialen und kulturellen Umgestaltung der Gesellschaft und ihrer Ausrichtung auf die Industrialisierung der Lebens-

35 Becker \& Langosch 2016.

36 Hettlage 1989, S. 300. 
mittelherstellung. ${ }^{37}$ In Zeiten der Wachstumskritik kann der Anthropologe Gudeman ${ }^{38}$ jedoch das Desinteresse der Wirtschaftswissenschaften gegenüber den Praktiken in Haushalten nur mehr als befremdend und kurios beurteilen, denn seine Forschungen zeigen deutlich, dass Subsistenzwirtschaft und Kapitalismus mindestens gleichberechtigte Formen des Wirtschaftens darstellen.

»I see economy by its institutions or spheres, ranging from the house to meta-finance. Each sphere secures and distributes wealth distinctively from sharing to risk-taking, although the spheres and distributional modes may be combined. The spheres might be likened to a division of labor. The power of capital as well as closely held knowledge and social relationships takes place within this structure. ${ }^{39}$

Es ist also an der Zeit, die längst vorliegenden wissenschaftlichen Erkenntnisse als Grundlage wirtschaftspolitischer Entscheidungen heranzuziehen und sorgsame Formen des Wirtschaftens ernst zu nehmen, von denen in Zeiten des peak soil ${ }^{40}$ wahrscheinlich das Überleben der Menschheit im 21. Jahrhundert abhängen wird. ${ }^{41}$

\section{Resilienz und Bricolage}

Die globale Beschleunigung von Transaktionen und die daraus resultierende Instabilität von Märkten haben in den letzten zwei Jahrzehnten Resilienz zu einem Schlüsselgriff der Organisationsentwickler*innen werden lassen. Parallel dazu nimmt der Begriff aber auch in der Psychologie und in den Umweltwissenschaften einen prominenten Rang ein, geht es doch um die allseits erwünschte Fähigkeit, mit unvorhersehbaren oder unberechenbaren Widerständen, Notlagen und Verlus-

\footnotetext{
37 Galbraith 1974.

38 Gudeman 2016, S. 2.

39 Gudeman 2016, S. 5.

40 Vgl. Politische Ökologie 119 (2010).

41 Vgl. Shiva 2008.
} 
ten zurecht zu kommen, ohne dass Gesundheit oder Betrieb Schaden nehmen. Forschungsprojekte aus unterschiedlichsten Disziplinen stellen daher die Frage, wie Resilienz herzustellen sei, oder wie und unter welchen Bedingungen man resilientes Verhalten lernen könne. Die Agrarsoziologin Ika Darnhofer ${ }^{42}$ konzipiert auf der Grundlage ihrer Forschungen über bäuerliche Familienbetriebe Resilienz im Rahmen einer relationalen Soziologie und mit Rückgriff auf die von Gilbert Simondon ${ }^{43}$ beeinflussten Theorien des Philosophen Gilles Deleuze als ein "Werden ${ }^{44}{ }^{44}$ Sie verabschiedet Perspektiven, die Resilienz bisher als festschreibbare und eventuell wiederholbare Lernprozesse einzugrenzen versuchten. Vielmehr eröffnet sie ein interdisziplinäres Spektrum an Herangehensweisen. Vor allem ihre Übertragung Claude LeviStrauss' Metapher vom "wilden Denken ${ }^{45}$ auf bäuerliches Wirtschaften erweist sich als äußerst hilfreich, um das komplexe und kreative Handeln mancher Betriebsführer*innen im ökologischen Landbau besser zu verstehen.

Im Rahmen eines »wilden Denkens«, das er dem »domestizierten« und ergebnisorientierten Denken der rationalen europäischen Wissenschaftler*innen entgegenstellte, verwendete Lévi-Strauss die Bezeichnung bricolage. Ein Begriff, der mit »Bastelarbeit« oder auch »Pfusch« $\mathrm{zu}$ übersetzen ist und daher eine gewisse Geringschätzung zum Ausdruck bringt, die aus heutiger Sicht als eurozentristische Überheblichkeit kritisiert werden muss. Der Kulturanthropologe hielt z.B. die kunstvollen Erzählstrukturen, die brasilianische Stammeskulturen aus der Verknüpfung mythologischer Bausteine entstehen lassen, nicht für planvolles Vorgehen sondern für Bastelei oder mythopoietisches Schaffen. Als Strukturalist versuchte er aus den Relationen zwischen einzelnen Bausteinen der mythologischen Erzählungen kulturübergreifende Regeln zu abstrahieren, um allgemeine Gesetze für den Mythenbau festzustellen. Als ihm das aber nicht zufriedenstellend gelang, bezeichne-

\footnotetext{
42 Darnhofer u.a. 2016, S. 116.

43 Simondon 2007, S. 29-45.

44 Iliadis 2013.

45 Lévi-Strauss 1989.
} 
te er diese mythopoietischen Gebilde als unvorhergesehene Ergebnisse von Improvisationen. Das heißt, er erkannte kein strategisches, regelgeleitetes Vorgehen darin, sondern nur ein Experimentieren auf gut Glück ${ }^{46}$ - eine Vorgangsweise, die in Europa damals wie heute nicht besonders viel Anerkennung findet. Das »wilde Denken«, das er den Bororo oder den Nambikwara im Amazonasgebiet zuschrieb, ist nicht nur von rationalen Absichten geleitet, sondern vermischt ständig rationale Vorhaben mit Kompromissen oder Umwegen, die aus Gründen gewählt werden, die sich jeder Verallgemeinerung entziehen: Neugier, Spaß am Tun, Lust an Varianzen, Inspiration durch aktuelle Ereignisse, Wetterlagen oder Notfälle, was auch immer. Der Bastler oder die Bastlerin passt seine oder ihre Pläne an die Möglichkeiten und Ressourcen an, die zur Verfügung stehen und geht mit beschränkten Materialien kreativ um. ${ }^{47}$ Solche Improvisationen sind auch für kleinbäuerliche Biobetriebe mit beschränkter technologischer Ausstattung typisch. ${ }^{48}$ Die von uns untersuchten Betriebsführer*innen mussten sich das Know-How über Tiere, Pflanzen oder Käseerzeugung sowie die Anbaumethoden in Auseinandersetzung mit den örtlichen Gegebenheiten und der vorhandenen oder nicht vorhandenen betrieblichen Ausstattung auf sehr individuellen Wegen erwerben. Sie waren von Anfang an Bastler*innen einer Existenz, die so weder von ihnen selbst, ihren Herkunftsfamilien noch von den Behörden vorher angedacht war. Im Gegenteil: Viele Schwierigkeiten entstanden dadurch, dass behördliche Auflagen an nicht mehr praktikablen oder agrarindustriellen Vorstellungen von Tierhaltung und Pflanzenzucht festhielten und Innovationen gegenüber hinderlich wirkten.

Auch die Beziehungen zu anderen Bauern und Bäuerinnen wurden nicht immer positiv geschildert. Manche konventionell wirtschaftende Betriebsführer*innen halten ökologische Betriebsideen für unrentabel und verweigern auch den erfolgreichen Betrieben Anerkennung. Das äußert sich in abfälligen Bemerkungen und Witzen

47 Bies 2014, S. 212.

48 Darnhofer 2016, S. 118; Darnhofer 2014. 
unter den Männern im Dorf. Diese skeptischen Nachbarn führen selbst meist die seit den 1950er Jahren von den Landwirtschaftskammern der Landjugend regelrecht dogmatisch eingehämmerten Praktiken zur Effizienzsteigerung ${ }^{49}$ weiter. Da das Risiko eines Umstieges ins ökologische Wirtschaften tatsächlich hoch ist, beobachten sie eine ökologisch ausgerichtete Betriebsführung nicht nur mit Neugier, sondern fürchten manchmal auch die In-Frage-Stellung ihrer eigenen Entscheidungen, die dem Zwang zum Wachstum geschuldet sind. Die Tatsache, dass ökologische Betriebe oft so lange wie möglich mit alten Maschinen weiterarbeiten und in ihrer technologischen Ausstattung bewusst auf einem sehr niedrigen Niveau verharren, führt hin und wieder zu verächtlichen Blicken von Anrainern, die nur die Anschaffung neuer Maschinen als Zeichen des Erfolgs lesen können. Besondere Fähigkeiten, die etwa zum Einsatz modernster digitaler Medien im Kundenkontakt und bei der Vernetzung mit gleichgesinnten Expert*innen für Landbau führen, werden dabei übersehen. Die Betreiber"innen ökologischen Landbaus müssen es aushalten, in ihren Dörfern von einigen Mitbewohner"innen als Außenseiter*innen betrachtet und entgegen aller Evidenz als nicht ernst $\mathrm{zu}$ nehmende oder sogar realitätsferne Menschen beurteilt zu werden. Das macht ein Leben und Wirtschaften auf dem Land nicht leichter. Obwohl die Landwirtschaftskammer das »neue Leben auf dem Land« fördern will, verbreitet sie in der Beratung dennoch häufig wirtschaftsrationale Betriebskonzepte. Selbst wenn Vertreter*innen der Kammer ökologischen Landbau oder CSA-Konzepte als Lösung für betriebliche Probleme empfehlen, so geschieht dies im Kontext der klassischen Betriebswirtschaftslehre, die Trends aufnimmt, ohne das effizienzorientierte Weltbild zu ändern. Der "Geist des Kapitalismus ${ }^{50}$ bleibt einstweilen in diesen bäuerlichen Organisationen der vorherrschende. ${ }^{51}$ Welche Eigenschaften und Ressourcen es aber für

49 Vgl. Kaser u.a. 2003, S. 329-338.

50 Vgl. Boltanski \& Ève 2003.

51 Die Autorin wurde z.B. für eine Weiterbildungsveranstaltung von Bäuerinnen eingeladen. Nachdem sie den Veranstalter*innen das Konzept ihres Vortrags 
sorgsame Landwirtschaft braucht, bzw. welche Persönlichkeiten und Kommunikationsfähigkeiten nötig sind, wissen institutionell tätige Berater*innen nur selten. Sorgsame Landwirtschaft ist kein Konzept, das sich in einer Broschüre zusammenfassen oder in Workshops unterrichten ließe. Alle befragten Betriebsführer*innen müssen knapp kalkulieren, dennoch kommt in den Gesprächen klar zum Vorschein, dass Geld in einem Bündel an Motivationen zwar eine bedeutende Rolle spielt, jedoch den Rahmen der persönlichen Weltanschauung nicht sprengen darf. Die stärksten Motive entspringen einem Weltbild und persönlichen Erfahrungen, die man in der Beratung nicht einfach weiterreichen kann. So gesehen bräuchten die "neuen Bauern und Bäuerinnen« auch neue Institutionen, die nicht auf den Erhalt alter Strukturen und jener Vorstellungen vom bäuerlichen Leben fixiert sind, die sich in den 1960er Jahren etabliert haben. Immerhin - so manifestiert sich ein "wildes Denken« - haben sie sich zu vernetzen gelernt. Da die herkömmlichen Fachberatungen der Landwirtschaftskammer ihnen wenig Neues vermitteln können, haben sie begonnen, selbst Netzwerke aufzubauen, die aber regionsübergreifend und z.T. auch international sind. Die erfolgreichen Betriebsführer*innen haben Praktika, Auslandsreisen und verschiedene berufliche Experimente hinter sich und sind den Behörden im Wissenserwerb bezüglich ökologischer Landwirtschaft weit voraus. Dieser Umstand bestätigt die Hypothese, dass Beziehungen Innovationsprozesse fördern, gleich ob es sich um Beziehungen $\mathrm{zu}$ Menschen oder $\mathrm{zu}$ nicht-menschlichen Lebewesen oder sogar zu Dingen handelt. Beziehungen erzeugen Wertschätzung und fördern die Generierung von neuem Wissen, das aus unpersönlichen Top-down-Strukturen und Lehrbüchern ohne interdisziplinärem Ansatz nicht gewonnen werden kann. Innovationen entstehen eben

mit Kritik an der Werbekampagne »Genusslandschaft « zugesandt hatte, wurde sie wieder ausgeladen. Das Marketing wird von Kammerbeiträgen teuer bezahlt, doch das Etikett »Cenusslandschaft« stellt keine kulturellen oder sozialen Beziehungen zur Region her, sondern setzt von Werbefachleuten am Schreibtisch erfundene Vertriebskonzepte um, die am Megatrend Erlebniskonsum ausgerichtet sind. 
immer durch »Bastelei«, das heißt durch nicht unbedingt zweckrationale Verknüpfung von Faktoren und durch das Verlassen der alten ausgetretenen Wege. Ein funktionierender ökologischer Kleinbetrieb ist gefordert, sich ständig anzupassen: Dem Wetter, den gesetzlichen Vorgaben, den wechselnden Interessen der Kund*innen und den Bedürfnissen der Mitarbeiter*innen. Diese Anpassung stellt jedoch keine Unterwerfung dar, wie sie das globale Nahrungsregime fordert, sondern sie entspringt einer inneren Bereitschaft mit der Umwelt in Beziehungen zu treten.

\section{Sorgsamkeit, relationale Güter, Lebens-Mittel}

Das Wörterbuch der Gebrüder Grimm unterscheidet Sorge von Sorgsamkeit. $^{52}$ Es beschreibt Sorgsamkeit als eine spezifische Qualität, die verschiedenste Tätigkeiten auszeichnen kann. Sorgsames Handeln stellt eine Vorbeugung ernster Sorgen dar. Somit ist Sorgsamkeit nichts das bedrückt oder belastet, sondern hat im Gegenteil noch mehr als die Sorge mit dem guten Leben zu tun. Sorgsamkeit impliziert eine Haltung der Aufmerksamkeit und Offenheit gegenüber Mitmenschen und Umwelt.

Der Sorgecharakter vieler biobäuerlicher Tätigkeiten rückte erst im Verlauf der Erhebungen und der Auswertung der Interviews ins Blickfeld. Vielfältige Austauschprozesse verbinden einzelne Menschen mit Gemeinschaften und ökologischen Systemen, sodass eine fürsorgliche Praxis rekonstruiert werden kann, welche die Unterscheidung zwischen Natur und Kultur oft aufhebt. Die Interviewpartner*innen selbst empfinden das auch so. Sorgsamkeit überschreitet die Grenzen zwischen den Spezies. Gerade in Biobetrieben muss oft entschieden werden, ob man dem Boden, den Pflanzen oder den Tieren auf Kosten des finanziellen Gewinnes mehr Zeit schenkt. Bei jeder Investition muss abgewogen werden, inwiefern sie sich nicht nur auf der ökonomischen sondern auch auf der ökologischen und emotionalen Ebene lohnen wird, oder 
ob das ökonomische Kalkül etwa dem Wohlbefinden der Tiere oder der Qualität der Früchte nachgereiht werden sollte. Die interviewten Akteur*innen äußerten solche Überlegungen häufig, denn sie verdinglichen weder die tierischen und pflanzlichen Lebewesen noch die Menschen, obwohl die Entscheidungsspielräume knapp sind. So wie die für diese Studie interviewten Personen sich und ihre Arbeit beschreiben, müssen wir sie als intrinsisch motiviert bezeichnen. Sie wollen nicht in erster Linie nur Geld verdienen, sondern sie wollen mit sinnvoller und selbstbestimmter Arbeit ihren Lebensunterhalt bestreiten und dabei ein gutes Leben haben. Das bedeutet, dass sie die nicht-menschlichen Lebewesen mit Respekt behandeln, dem die Effizienz nachgereiht wird. Soziologische Studien haben bäuerliche Sozialstrukturen oft dichotomisch den urbanen gegenübergestellt und den ersteren Irrationalität und traditionales Denken, letzteren hingegen Rationalität und technikaffines Denken unterstellt. ${ }^{53}$ Sich der Marktrationalität zu widersetzen und mit weniger als 20 Hektar seine Produkte in Konkurrenz zum Weltmarkt absetzen zu wollen, könnte durchaus als irrational bezeichnet werden. Doch Rationalität hängt davon $a b$, in welchem System man sich beheimatet fühlt. Ganz anders als die Soziolog*innen konnten Kulturanthropolog"innen die eher ablehnenden Haltungen gegenüber Neuerungen in bäuerlichen Gesellschaften als durchaus rational in Bezug auf Wertesysteme einstufen, die Gewinnmaximierung nicht an die erste Stelle setzten. ${ }^{54}$ Der Behauptung, dass Menschen aus bäuerlichen Strukturen beharrlich am Althergebrachten festhalten würden, stellten sie sogar die Beobachtung entgegen, dass sich Mitglieder einfacher bäuerlicher Gesellschaften durch eine besondere Experimentierfreudigkeit auszeichnen. ${ }^{55}$ Denn im relationalen Weltbild von Subsistenzbäuer*innen sehen Experimente anders aus, als moderne Wissenschaftler"innen das erwarten würden. »The traditional agriculturalist subsists not merely in a complex environment, he contribu-

\footnotetext{
53 Hettlage 1989, S. 299.

54 Hettlage 1989, S. 300.

55 Johnson 1972.
} 
tes much of that complexity through his own labor. « ${ }^{56}$, formulierte der Anthropologe Allen Johnson im Hinblick auf die fast intime Verbundenheit armer Menschen mit dem Boden und den Pflanzen, die ihr karges Überleben sichern sollten. Aus heutiger Sicht erkannte Johnson damals die komplexen Beziehungen zwischen Landbearbeiter*innen und ihrer Umwelt und verlangte Wertschätzung für diese Art der Sensibilität, statt sie nur als vormodern und irrational einzustufen. Vielmehr müsste man marktrational agierende Bauern und Bäuerinnen der Gegenwart als einfallslos bezeichnen, wenn sie außer der Vergrößerung der Betriebe und dem Einsatz immer größerer Maschinen keine neuen Ideen haben. Tatsache ist jedoch, dass das Diktat des Marktes, dem sie sich durch die Aufnahme hoher Kredite unterworfen haben, kaum neue Ideen zulässt. Sorgsame Landwirt*innen haben sich zur rechten Zeit gegen einengende finanzielle Abhängigkeiten und für mehr Rücksicht auf die Natur entschieden. Der Begriff der sorgsamen Landwirtschaft scheint geeignet, um diese besondere Relationalität zu beschreiben, die die so genannten »neuen Bauern und Bäuerinnen« durch Umsicht, Rücksicht und Voraussicht herstellen. Aus rein wachstums- und gewinnorientierter Sicht könnte ihnen zwar vorgeworfen werden, dass ihr Arbeitsaufwand $\mathrm{zu}$ hoch und die Gewinne zu klein seien. Allerdings ignoriert diese Rechnung, dass ökologische Betriebe sinnvolle Arbeit anbieten und dem Gemeinwohl keine schädlichen Nebeneffekte wie erhöhten $\mathrm{CO}^{2}$-Ausstoß und Pestizide aufbürden. Reich wird man wahrscheinlich nicht. Der Arbeitsaufwand und die Risiken sind tatsächlich hoch. Wenn Spätfröste z.B. zwei Jahre hintereinander die Obsternte vernichten, dann kann das den Betrieb stark belasten, notfalls müssen die Betriebseigner*innen etwas dazuverdienen. Und wenn die Kund"innen Äpfel mit kleinen Schorfflecken nicht mehr akzeptieren wollen, kann das zu jährlichen Einbußen führen, auf die dann kreativ reagiert werden muss. Durch gezielte Werbung in der Region oder Angebote an Schulen, wo man den hervorragenden Geschmack der Früchte ins Bewusstsein bringt, steigt der Absatz vielleicht wieder. Dazu braucht es viel Kreativität und Flexibilität. Der Gewinn sammelt sich außerdem 
nicht nur bei den Bauern und Bäuerinnen, sondern manifestiert sich in der Bodengesundheit, dem Tierwohl und der hohen Qualität der Nahrungsmittel, die nicht teurer verkauft werden als Bioprodukte von Eco-entrepreneurs ${ }^{57}$ (siehe Kapitel Kosnik). Und zudem kann ein CSABetrieb auch als Zentrum eines Netzwerks fungieren, in dem man neue Menschen kennenlernt. Denn im Unterschied zur industriellen Landwirtschaft, wo die Böden immer unfruchtbarer, die Maschinen immer größer und die Menschen, die sie steuern, immer einsamer werden, bereichert sorgsame Landwirtschaft die Region durch positive Externalitäten. ${ }^{58}$ Dennoch erhält sie wie der größte Teil der Sorgetätigkeiten in dieser Gesellschaft weiterhin zu wenig Anerkennung.

Im Deutschen hat sich mittlerweile Care als Synonym für Pflegeoder Sorgearbeit eingebürgert, die entweder bezahlt oder unbezahlt durchgeführt wird. ${ }^{59}$ Joan Tronto und Berenice Fischer definierten den Begriff caring work in den 1990er Jahren mit Bezugnahme auf Karl Marx' »Reproduktionsarbeit«.

"In the most general sense, care is a species activity that includes everything we do to maintain, continue, and repair our world so that we may live in it as well as possible. That world includes our bodies, our selves, and our environment, all of which we seek to interweave in a complex, life-sustaining web. ${ }^{60}$

Tronto bezieht also ausdrücklich die Sorge um die Umwelt und die nichtmenschlichen Lebewesen mit ein. Feministische Debatten um die ungleiche Verteilung unbezahlter Arbeit zogen seit den 1960er Jahren präzise historische und soziologische Analysen der Hausarbeit

In Anlehnung an den Begriffentrepreneurial agriculture von Ploeg (2018b) für agrarindustrielle Lebensmittelproduktion.

58 Gorz 2004, S. 20. Gorz versteht darunter gelingende Selbstregulierung zwischenmenschlicher Beziehungen, die auf Empathie und Anerkennung beruhen. Sie ist nicht quantifizierbar und nicht austauschbar, stellt jedoch die Voraussetzung für profitable Produktionsstrukturen dar und wird unbezahlt von Unternehmen verwertet.

59 Winker 2015, S. 17.

60 Fisher \& Tronto 1990, S. 40. 
und der Pflegetätigkeiten nach sich, die Tronto $\mathrm{zu}$ der essentiellen Erkenntnis führten, dass Care mit Bedürfnisbefriedigung $\mathrm{zu}$ tun hat und immer Beziehungen stiftet. "Care is about meeting needs, and it is always relational. «" ${ }^{61}$ Die Analogien zwischen Pflegearbeit, Subsistenzarbeit und bäuerlicher Arbeit haben Bennholdt-Thomsen u.a. schon vor vier Jahrzehnten herausgearbeitet. ${ }^{62}$ Sorgsame Landwirtschaft ist sowohl reproduktiv als auch produktiv und führt vor Augen, wie gefährlich diese Unterteilung ist. Denn eine rein auf Produktion ausgerichtete Landwirtschaft zerstört auf Dauer die Grundlagen des Wirtschaftens durch negative Externalitäten (Zerstörung des Humus, Verunreinigung des Wassers, Bodenverdichtung etc.). Im sozialen Bereich erzeugt die Missachtung der Pflegearbeit als Quelle volkswirtschaftlichen Reichtums große soziale Ungleichheiten zwischen den so genannten produktiven und reproduktiven Branchen und vergrößert außerdem den Gender Pay Gap aufgrund der geschlechtsspezifischen Zuteilung unbezahlter oder schlecht bezahlter Pflegearbeit.

Bäuerliche Tätigkeiten waren besonders in der untersuchten Region der Oststeiermark bis in die 1960er Jahre mit einer starken Beziehung der Arbeitenden zu Erde, Tieren und sozialer Gemeinschaft verknüpft. Diese Gemeinschaft war außerdem oft zugleich die Gemeinschaft der Konsument*innen, denn Fleisch, Korn, Milchprodukte und etwa Kartoffeln wurden auch von den Familien selbst verzehrt. Dies trifft auch auf andere österreichische Regionen mit kleinbäuerlichen Betrieben und geringem Technologieeinsatz zu. Ziel der bäuerlichen Arbeit war der Erhalt des Betriebes und der Familie. Zwar hing der Erfolg vom Verkauf der Produkte ab, doch bei den meisten Tätigkeiten auf dem Hof standen vor der Vermehrung des Geldes der Betrieb selbst und das gute Leben der von ihm Abhängigen an erster Stelle. Eine Betriebsvergrößerung oder Ertragssteigerung wurde erst $a b$ den 1960er Jahren zwingend. ${ }^{63}$ Damals begann unter dem Druck zur Anpassung an Weltmarktbedingungen auch in den entlegenen Gebieten Ös-

\footnotetext{
61 Tronto 2015, S. 4.

62 Bennholdt-Thomsen u.a. 1983.

63 Vgl. Kaser u.a. 2003, S. 333.
} 
terreichs mit kleinen Betrieben die Spezialisierung der Landwirtschaft. Die Entwicklung komplexer Maschinen und pharmazeutischer Produkte sowie spezielle Tierzüchtungen ließen Investitionen nun auch bei kleinem Betriebskapital nicht nur rentabel sondern sogar überlebensnotwendig erscheinen. Kaser und Stocker ${ }^{64}$ zeigen - ebenso wie Müller für Deutschland - den Mentalitätswandel, der in der bäuerlichen Bevölkerung Österreichs in der 2. Republik parallel zur Abwanderung in die Städte stattfand. ${ }^{65}$ Die Forschungen von Heide Inhetveen und Mathilde Schmitt ${ }^{66}$ betonen aber eine doppelte bzw. widersprüchliche Mentalität: Einerseits wollen Landwirt*innen kleinerer Betriebe nicht völlig abhängig von den Marktmechanismen werden, andererseits entwickeln sie dennoch eine gutes unternehmerisches Gespür. Wie Andrea Heistinger in ihrer Analyse der Interviews ausarbeitet, so entwickeln sich sorgsame Einstellungen aus den je einmaligen Lebenserfahrungen der einzelnen Personen. Sie treten nicht zufällig auf, sondern wurzeln in spezifischen familiären Erlebnissen und Strategien aus der Vor- und Nachkriegszeit. Sie kommen also aus einem lebendigen Feld, das auf jede heranwachsende Generation einwirkt. Es ist ein Feld aus vielfältigen Beziehungen, das Umgangsweisen mit Krisen und die Resultate des Handelns speichert. Der Beschluss von Martin Bergmann, die profitorientierte und profitable Geflügelzucht des Vaters zu schließen und auf einen CSA-Betrieb mit Bioprodukten umzustellen, erfolgte z.B. nicht gegen die Entscheidungen seiner Vorfahren, sondern folgte in gewissem Sinn ihren wohlüberlegten Handlungsweisen. Denn die ökonomischen Bedingungen hatten sich geändert und dem jungen Mann war bewusst, dass er an einem Scheideweg stand. Das lebendige Beziehungsfeld aus dem heraus er seine Entscheidung traf, weist sowohl eine historische Dimension als auch die Dimension der Sorge auf. In beiden Dimensionen hoffen die Gebenden nicht auf eine Gegengabe. Die Eltern geben in dem meisten Fällen etwas an die Kinder weiter ohne Wunsch nach Bezahlung. Unbezahlt Sorgende rechnen zwar mit einem

64 Kaser \& Stocker 1987.

65 Vgl. Hofreither 1997, S. 641-651.

66 Inhetveen \& Schmitt 2010, S. 111-136. 
Nutzen, jedoch nicht (nur) für sich selbst, sondern für Mitmenschen, das Gemeinwohl oder die Mitwelt im weiteren Sinn inklusive nichtmenschlicher Lebewesen und ihrer nachfolgenden Generationen. Zwar handelt es sich um Reziprozitätsbeziehungen, jedoch geht es nicht um Verrechnung. Denn die Beziehungen sind intrinsisch motiviert und daher ad hoc lohnend. Die Bezugnahme auf ein »wir«, zu dem im Fall ökologischen Landbaus auch die zoologischen und botanischen Mitwesen zählen, erzeugt Beziehungen, die sich bei diesen Betrieben in der materiellen Qualität der Nahrung niederschlagen und die unverkäufliche Voraussetzung für Ernährungszufriedenheit bilden. Durch solche spezifischen sozialen Beziehungen und Interaktionen entsteht ein immaterielles "Drittes«, das der Soziologe Pierpaolo Donati als relationales Gut bezeichnet. ${ }^{67}$ Vorschriften, Auflagen oder materieller Zwang können dieses Gut nicht einfordern. Der Markt kann relationale Güter zwar verwerten aber nicht produzieren, sondern nur in der Werbung vortäuschen. Eine kluge Verknüpfung von Markt und staatlichen Maßnahmen kann die Entstehung relationaler Güter jedoch fördern. Denn sie stellen den besonderen Gewinn einer Region dar, in der sorgsame Landwirtschaft betrieben wird. Donati nennt folgende Bedingungen für ihre Entstehung: Die Teilnehmenden haben persönliche Kontakte und Interesse füreinander, sie achten auf die Bedürfnisse der Involvierten, die das relationale Gut nur miteinander herstellen und genießen können. Relationale Güter können nicht produziert werden sondern emergieren in einem Beziehungsfeld. Sie benötigen eine gewisse Dauer, weil Beziehungen keine punktuellen Phänomene sind, sondern historisch wachsen müssen. Wären keine Menschen beteiligt, spräche man von einem Ökosystem, einer Lebensgemeinschaft von unterschiedlichen Organismen und ihrer unbelebten Umwelt. Da aber Menschen und ihre kulturelle Dimension hinzukommen, sieht die Sympoiese anders aus. Sorgende Landwirtschaft schafft lebendige Felder des Austauschs, denen echte Lebens-Mittel entspringen, die materiell, sozial und symbolisch satt machen. 
In der sorgsamen Landwirtschaft stehen Menschen zwar in einem profitorientierten Austausch aber sie respektieren und unterstützen immer auch einen Gabenfluss, der im Gegensatz zu den sozial unwirksamen Deals des Marktes das gute Leben in seinen körperlichen, emotionalen und kulturellen Dimensionen garantiert. 
